\title{
New teaching focus of FLE to a new sociolinguistic context
}

\section{[Nouveau focus d'enseignement de FLE à un nouveau contexte sociolinguistique]}

\author{
Klementina Shiba
}

DOI: 10.18355/XL.2020.13.01.11

\begin{abstract}
As language learning is also about learning culture, this article proposes to address the issue of authentic FFL (FLE) teaching in a new sociocultural context where a teachercentred teaching is replaced by a learner-centred approach. Although the communicative approach, according to the psycholinguistic model, favours the learning of languages, we are in the conditions of teacher-centred teaching.During the teaching / learning of a language, one does not only teach language and lexicogrammatical models but adopts a new system of thought and knowledge about the culture, which requires a greater exposure to authentic communication contexts that simply didactic materials cannot offer. The human tendency to view technology in an anthropomorphic way increases the potential of the Internet to support the process of authentic language teaching/learning using ICT. Research and practice both support the interactive sociolinguistic model, which focuses primarily on the social aspect of language and the discovery of linguistic barriers in the communication process, thus orienting the authentic teaching of languages towards a learner-centered teaching of skills.
\end{abstract}

Key words: interaction, environment, ICT, sociolinguistics, authentic

\section{Résumé}

Comme l'apprentissage de la langue vise aussi l'apprentissage de la culture, cet article se propose d'adresser des questions de l'application de l'enseignement de FLE authentique à un nouveau contexte socioculturel où l'enseignement centré sur l'enseignant laisse la place à celui centré sur l'apprenant. Bien que l'approche communicative, d'après le modèle psycholinguistique, favorise l'apprentissage des langues, on est dans les conditions d'un enseignement centré sur l'enseignant. $\mathrm{Au}$ cours de l'enseignement /apprentissage d'une langue, on n'enseigne pas seulement la langue et des modèles lexico-grammaticaux, mais on adopte un nouveau système de pensée et de connaissances sur la culture. Cela nécessite une plus grande exposition des apprenants à de nouveaux contextes de communication authentiques, étant donné que les matériaux simplement didactiques ne peuvent pas l'offrir. La tendance humaine, à considérer la technologie de manière anthropomorphique, augmente le potentiel d'Internet pour soutenir le processus d'enseignement /apprentissage authentique des langues à l'aide des TIC.

Ainsi des recherches et de la pratique soutiennent également le modèle sociolinguistique interactif, qui met l'accent en premier lieu sur l'aspect social de la langue et la découverte des barrières linguistiques dans le processus de communication orientant l'enseignement authentique des langues vers un enseignement de compétences centré sur l'apprenant.

Mots-clés : interaction, environnement, TIC, sociolinguistique, authentique

\section{Introduction}

Grâce à l'intérêt des générations pour la nouveauté, les technologies de l'information et de la communication (TIC) ont impliqué tous les secteurs de la vie

XLinguae, Volume 13 Issue 1, January 2020, ISSN 1337-8384, eISSN 2453-711X 
jouant un rôle très puissant dans la société, influençant la façon dont les gens communiquent, s'informent, se forment, travaillent et s'amusent. Sur le plan social ces technologies sont considérées essentiellement comme une espace de divertissement et un moyen de prise d'information mais de plus en plus il s'est accentué leur grand potentiel/ contribution dans divers domaines professionnels tel que dans le domaine de l'enseignement/apprentissage en général et en particulier à celui des langues étrangères. La langue n'est plus considérée comme « un système, » mais comme «moyen de communication linguistique, culturel et social » (CECRL, 2001: 16).

Le partage de l'information et la motivation à la collaboration peuvent-ils être des facteurs de succès pour l'enseignement/apprentissage des langues ?

Cette étude est une contribution à la didactique des langues étrangères, au cours de son développement dans de nouveaux environnements d'enseignement en présence de nouvelles technologies de l'information et de la communication (NTIC).

Dans le processus d'enseignement/apprentissage le rôle traditionnel de l'enseignant a changé et l'apprenant devient collaborateur ayant plus de responsabilités dans la réalisation de la tâche et bénéficiant un degré plus élevé d'indépendance. Ainsi, l'apprenant est considéré comme « un acteur social qui sait réaliser les taches » (CECRL, 2001: 16).

En présence de ce nouveau contexte sociolinguistique on pense aux stratégies qu'il faut suivre de la part de l'enseignant et à son impact à l'apprentissage en général et plus particulièrement à celui des langues étrangères. Les TIC font déjà partie de la vie des étudiants, alors c'est le rôle des chercheurs et des institutions de recherches qui doit les faire entrer en jeu afin d'en profiter en les incorporant dans les programmes et le processus d'enseignement devenant TICE.

Pour cette raison il est nécessaire de définir de nouvelles stratégies de l'enseignement, afin de les introduire à l'apprentissage en général et précisément à celui d'une langue étrangère. Dans l'enseignement des langues étrangères, il y a de nombreux éléments qui influencent la performance des enseignants et des apprenants. $\mathrm{Au}$ cours de ce processus, de nombreuses techniques et de nouvelles stratégies orientées et dirigées par l'enseignant peuvent être utilisées en vue de motivation et implication des apprenants pour qu'ils réussissent à une communication interculturelle supposée. CECRL a marqué un pas important dans le processus de l'enseignement /apprentissage des langues étrangères, en apportant des changement sur le "paradigme de la pédagogie moderne »: le centre d'intérêt est déplacé vers les besoins communicatifs de l'apprenant, l'utilisateur de la langue.

En fait les TICE créant une espace de communication interactive peuvent être utilisées comme un outil parallèle, un support pédagogique et une source pour renforcer le contenu du cours de l'enseignement/apprentissage des langues, pour le rendre plus efficaces et plus attrayants afin d'assurer de hauts niveaux de la motivation, du succès à l'apprentissage des langues et apprendre à mieux apprendre.

Les TIC et l'enseignement/apprentissage des langues étrangères ne se sont pas inconnus les uns des autres. Rappelons les laboratoires des langues étrangères installés dans les années soixante, dont l'utilisation s'est vite réduite à l'arrivée des ordinateurs, rapidement mis au contrôle par les étudiants, perçus dès le début comme des outils favorables à l'apprentissage des langues, dont les logiciels didactiques ont eu, en grande partie, un caractère autodidacte, mettant donc en évidence leur caractéristique autonome à l'apprentissage. " [...] le domaine de l'enseignement des langues assisté par ordinateur n'ait cessé de se développer pour devenir, à l'heure actuelle, un domaine de recherche assez important au sein de la linguistique appliquée en général, et dans le domaine de la didactique des langues en particulier.» (Desmet, 2006: 119)

Les changements spectaculaires dans l'apprentissage des langues par l'arrivée de l'ordinateur ont mis en évidence l'importance de l'interaction humaine 
favorisant la communication, du développement des savoir-faire, de la création de la compétence d'une réflexion critique, de l'apprentissage autonome continu où l'enseignant doit guider les apprenants à interagir dans les nouvelles communautés virtuelles en utilisant un discours approprié. «La communication est rendue économique et efficace par la supposition réciproque faite par les participants à une situation donnée que des savoirs sont partagés concernant le code linguistique, les usages sociaux, les savoirs quotidiens sur le monde. » (Bange, 1996:189)

A l'ère de la mondialisation il faut lier le socioculturel à l'environnement concret des étudiants vu que l'enseignement/apprentissage des langues étrangères vise une formation en matière de communication sociale à travers une stratégie de l'immersion interculturelle. "Ces recherches fondées sur une prise de position reflètent l'état des relations entre l'enseignement/apprentissage et les conditions sociales où elles sont ancrées. 》 (Byram, 2011: 256)

Les technologies numériques (TN) considérés essentiellement comme un moyen de prise d'information ou un espace de divertissement ont une grande contribution positive dans divers domaines professionnels tel que dans l'enseignement en général et particulièrement dans celui des langues étrangères. "Cette montée en puissance du numérique n'a pas manqué d'affecter le secteur de l'enseignement, celui des langues en particulier [...]. » (Desmet, 2006:119)

Cette étude aborde des réflexions basées sur ma propre expérience récente, portant de l'hypothèse suivante: La création d'un nouvel environnement d'enseignement/apprentissage en présence des TIC, élevant le degré et la qualité d'exposition à de nombreuses contextes linguistiques authentiques, créant un nouveau contexte sociolinguistique et culturel, un espace d'interaction des enseignants et des apprenants dotés de nouvelles représentations, est une contribution positive à l'enseignement/apprentissage du FLE authentique. Cette hypothèse repose sur les questions suivantes :

- $\quad$ En quoi les TICE contribuent positivement au processus de l'enseignement/apprentissage de FLE authentique?

- L'enseignant, joue-t-il un rôle dans l'orientation des apprenants et l'accélération du processus de l'apprentissage en vue d'en profiter une meilleure performance?

La mise en œuvre des TIC dans le domaine de l'enseignement des langues étrangères est en tendance ascendante, bien que cela ait suscité beaucoup de débats sur les formes concrètes et les contextes particuliers de leur implication. "Ceci explique pourquoi les (N)TIC font de plus en plus partie des outils de base de tout enseignant de langue, et pourquoi la classe de langues moderne devient, au moins en partie, numérique. » (Desmet, 2006:119)

Comme l'utilisation des TIC a été discrètement inclue dans le mode de vie et la pratique quotidienne, elles ont également amélioré les méthodes d'enseignement, innovant des approches pédagogiques efficaces en incluant de nouvelles stratégies pédagogiques en vue d'aider les étudiants dans le processus de l'apprentissage en leur assistant à leurs besoins et à l'évolution de leur perception sur leurs capacités à apprendre.

A ce stade de l'enseignement/apprentissage des langues, les enseignants doivent viser de "Différencier la pédagogie pour mieux lutter contre l'échec scolaire; rénover les contenus et les didactiques; développer les pédagogies actives, participatives, coopératives; [...].»(Perrenoud,1994: 9)

Compte tenu de la manière dont les langues sont apprises, de nombreux enseignants et apprenants, exposés quotidiennement à Internet et aux nouvelles technologies de l'information et de la communication, qui ont

XLinguae, Volume 13 Issue 1, January 2020, ISSN 1337-8384, eISSN 2453-711X 
fortement influencé l'enseignement/apprentissage par leurs moyens, en ont beaucoup profité en y introduisant de nouvelles stratégies et techniques en vue de réussir à l'apprentissage de FLE. L'expérience pédagogique montre que «La technologie numérique offre des avantages évidents pour atteindre les objectifs ambitieux de l'enseignement.»l (Turkle, $1996: 12$ )

L'enseignant guide les apprenants à interagir dans les nouvelles communautés virtuelles en utilisant un discours approprié. "Le but essentiel de l'enseignement dans des environnements en ligne est l'interaction. $»^{2}$ (Gilbert, \& Moore, 1998: 29)

Dans l'enseignement des langues étrangères, parmi de nombreux éléments qui influencent la performance des enseignants et la réussite des apprenants, les TICE s'utilisent en fonction de l'interaction à l'amélioration des buts de l'enseignants visés à travers de diverses stratégies. "Cependant, on a vu émerger ces dernières années des outils qui rendent possible l'interaction parlée entre l'utilisateur et l'ordinateur ou qui facilitent la communication entre deux personnes par le biais de l'ordinateur. » (Desmet, $2006: 121)$

\section{- En quoi les TICE contribuent positivement au processus de l'enseignement/apprentissage de FLE authentique?}

Plusieurs recherches pédagogiques et sociolinguistique soutiennent l'idée que l'utilisation des TIC à des fins d'enseignement/apprentissage des langues, présente plus d'avantages que d'inconvénients. La présence d'une «communauté en ligne» est une aide à l'apprentissage de FLE qui se réalise en collaboration de trois éléments fondamentaux: la présence sociale ; la présence enseignante ; et la présence cognitive. Ce type d'apprentissage en dehors de la salle de classe se caractérise d'une forte motivation, d'un grand plaisir à apprendre, d'un apprentissage continu, autonome de langue authentique, et de la familiarité des étudiants au cours de ce processus à travers une interaction significative étudiant-enseignant et étudiantétudiant.

Les TIC permettent aux apprenants d'interagir avec l'enseignant et les uns les autres à cet environnement hybride d'enseignement ; en salle de classe et en ligne, ce qui affecte la motivation des apprenants, prenant de la responsabilité vis à vis des tâches. Les apprenants renforcent des pratiques au service de l'apprentissage tel que la vision, l'écoute, la lecture, l'écriture dû au fait qu'ils explorent en ligne une vaste ressource de documents authentiques écrits, audio, vidéo à travers lesquels ils créent et appliquent des compétences réceptives et productives, composants importants $d u$ processus d'apprentissage d'une langue étrangère, qui conduit à un auto-apprentissage continu. "Au lieu d'absorber des connaissances, l'apprenant les construit, [...]. $L$ 'apprenant n'a acquis ces connaissances que s'il est en mesure de les appliquer. D'où l'importance fondamentale de la pédagogie de la tâche et de l'enseignement axé sur les compétences.» (Desmet, 2006 : 127)

Les recherches récentes et les applications actuelles dans le domaine de l'enseignement ont modifié même l'orientation théorique en remettant en question l'ancien modèle en faveur du modèle sociolinguistique interactif qui souligne, en premier lieu, l'aspect social de la langue et la découverte des barrières linguistiques dans le processus de la communication, en orientant l'enseignement des langues

\footnotetext{
${ }^{1}$ La traductions est faite par moi ( Shiba, K).

${ }^{2}$ La traductions est faite par moi ( Shiba, K).
} 
étrangères vers un enseignement de création de compétences centré sur l'apprenant. «Les compétences s'apprennent et se développent dans divers contextes :

- contexte éducatifformel : l'école

- contexte éducatif non formel: la famille, les lieux de travail, les massmédia, les organisations culturelles, associatives

- contexte éducatif informel: la vie social.» (Licheri, 2018:11)

Les TIC, multipliant le degré et la qualité d'exposition à de nombreuses contextes linguistiques authentiques, soutiennent fortement l'enseignement /apprentissage de FLE authentique en facilitant les enseignants à communiquer avec leurs étudiants en dehors de la salle de classe, à discuter sur les devoirs, à leur envoyer des corrections et les apprenants à partager leurs intérêts, publiant, téléchargeant de différentes services pour qu'ils aient de nombreuses informations utiles autour d'un sujet précis. "Cette méthodologie place l'apprenant au centre de son apprentissage. Cela va changer le rôle de l'enseignant en classe de langue. Il doit favoriser les interactions entre les apprenants et leur proposer des situations de communication stimulantes. » (Hirschsprung, 2005:14)

\section{- L'enseignant, joue-t-il un rôle dans l'orientation des apprenants et l'accélération du processus de l'apprentissage en vue d'en profiter une meilleure performance?}

Le nouveau rôle de l'enseignant comme leadership et pas une simple source de connaissances dans la salle de classe, est très important voire décisif dans l'organisation du cours en orientant l'apprenant à la recherche de divers documents authentiques dans de différentes ressources. "L'apprentissage, l'acquisition et la maitrise de différents aspects des langues et cultures ont touché des questions auxquelles il faut répondre avec un enseignement pertinent, diffèrent, équilibrée pour l'apprenant et pour la matière enseigné aussi » (CECRL, 2001: 16).

La tendance des individus à interagir avec les ordinateurs de manière fondamentalement sociale rend possible la conciliation, la promotion et le développement de ces deux environnements d'enseignement : en classe et en ligne. Le rôle de l'enseignant est d'essayer, à l'aide des TIC, de concilier de divers environnements de l'enseignement/ apprentissage à travers l'interaction dans le but d'améliorer les résultats de l'apprentissage de FLE. "La tendance humaine à considérer la technologie de manière anthropomorphique comme une sorte d'aide personnelle avec laquelle elle coopère à atteindre un objectif, augmente le potentiel d'Internet pour soutenir le processus d'apprentissage des langues étrangères.» ( Blake, 2008:19)

Il faut noter que l'interaction visant la compréhension, qui joue un rôle majeur dans l'apprentissage, ne rend pas possible une acquisition linguistique «instantanée», cela vient progressivement, avec le temps et par la suite d'un nombre d'interactions au moyen des TICE d'où la mission de l'enseignant à promouvoir, faciliter et développer cette forme d'enseignement non isolé et le rendre efficace. "Qu'on le veuille ou non, la place de l'enseignant ne cesse d'évoluer. C'est ainsi que même dans le contexte du groupe-classe, l'enseignant devient un passeur, celui qui agence le va-et-vient entre l'apprenant et les ressources. " (Valenzuela, 2010: 83)

L'utilisation de ces technologies est introduite dans le programme d'études de FLE grâce à la mise en œuvre des formes diversifiées d'enseignement par la pédagogie de projet favorisant l'interaction en ligne d'où le rôle stimulant des TIC.

${ }^{3}$ La traductions est faite par moi ( Shiba, K).

XLinguae, Volume 13 Issue 1, January 2020, ISSN 1337-8384, eISSN 2453-711X 
"Pour une mise en cuvre pratique plus significative et plus productive de la théorie du constructivisme social en classe, plusieurs éléments clés sont nécessaires pour orienter au mieux la conception des programmes. » (Powell \& Kalina, 2009: 239) ${ }^{4}$

Dans ce nouveau contexte, les représentations de l'enseignant, en tant que leader, sur la façon d'enseigner le FLE iraient changer pour soutenir et encourager en pratique l'apprenant à utiliser activement ces nouveaux outils pour comprendre et effectuer l'activité concernée. " Du même coup, c'est aussi le rôle de l'enseignant qui évolue. Traditionnellement, celui-ci était un «narrateur omniscient (instructeur), détenteur et dispensateur des connaissances requises.» (Desmet, 2006:124)

A travers une planification, l'enseignant propose un enseignement répondant aux besoins des apprenants, de la manière dont ils le veulent et préfèrent, visant un apprentissage durable et une assimilation optimale effective du FLE par étapes successives bien définies, présentées en détail, en vue de la création des compétences et de l'indépendance de la pensée de l'apprenant. L'apprenant n'est plus considéré comme un simple récepteur passif des instructions de l'enseignant, au contraire, les apprenants interagissent mutuellement en partageant les connaissances et la responsabilité des tâches, ce qui entraîne un changement du focus de l'enseignement vers un enseignement centré sur l'apprenant et la construction des connaissances et des compétences. "De toute évidence, il s'agit d'un contexte typique d'enseignement centré sur l'apprenant, où l'accent se met sur la nature communicative, le dialogue social et la construction des connaissances. » ( Powell \& Kalina, 2009: 243)

La coopération des étudiants entre eux dans un tel espace, au moyens des TIC, constitue un mécanisme puissant d'acquisition, permettant le partage de rôles et un accès continu à des formes de contrôle conformément aux exigences de la tâche, sans négliger les étapes et les éléments du processus de l'enseignement /apprentissage classique, ce qui nous permet de faire des parallèles avec la salle de classe en tant qu'environnement d'apprentissage linguistique et culturel. «En outre, le e-learning se concentre encore en priorité sur des domaines de connaissances comme le lexique ou la grammaire,[...]domaines jusqu'à présent réservés de préférence au c-learning.» (Desmet, 2006: 129)

L'enseignant, qui dirige en tant que facilitateur ce processus, met les apprenants dans des relations réciproques continuelles, auxquelles chacun y participe en ajoutant de la valeur et du sens à la communication, en évaluant et en organisant les informations mentalement. "De nos jours, l'enseignant de langues n'est plus le détenteur, le diffuseur de tous les savoirs ; il est devenu "conseiller et un organisateur" des apprentissages. " (Valenzuela, 2010 :89)

Selon la théorie du constructivisme social, qui a servi de base au développement de ces nouvelles modèles pédagogiques, l'information est construite initialement lors de l'interaction sociale pour être ensuite traitée à l'intérieur par l'individu, respectant la manière et le rythme dont chacun intériorise l'information. "La nouvelle génération des multimédias met à notre disposition des didacticiels développant l'autonomie et la compétence communicative de nos apprenants, en individualisant les taches, les contenus et permettant à chacun de travailler à son rythme.» (Biggs, 1997:10)

La création d'un environnement d'enseignement centré sur l'apprenant est également le principe de base de la théorie du constructivisme social. L'apprenant devrait découvrir et créer un sens à partir de l'information. L'enseignant pour augmenter la motivation et la confiance en soi de l'apprenant, doit prendre en compte le contexte social dans lequel un problème interagit, dont l'exposition devrait inclure les étudiants afin qu'ils soient capable d'interagir de manière critique et de l'influencer.

Ainsi on transforme cette "classe » d'apprentissage à caractère social, en une communauté d'interaction comme une réalité essentielle et nécessaire pour cet

${ }^{4}$ La traductions est faite par moi ( Shiba, K). 
environnement de l'enseignement/apprentissage qui aborde de nouveaux rôles pour ses acteurs: enseignants en tant que leaders, facilitateurs et étudiants en tant qu'apprenants autonomes. "L'autonomie n'est pas un processus simple, partagé par tous. Elle suppose une capacité mentale à prendre de la distance par rapport à ses propres démarches, à analyser et formaliser ses propres stratégies afin de les optimiser et les gérer de façon indépendante.» (Aycan, 2013: 87)

L'apprentissage authentique est lié au fait que le problème et la situation ne devaient pas être considérés hors contexte, car ils fournissent des données nécessaires que l'apprenant devrait évaluer lors de son analyse. Les étudiants en cours d'apprentissage ne se contentent pas à l'apprentissage d'une langue ou bien des modèles lexico-grammaticaux, mais ils adoptent un nouveau système de pensée et avant tout la connaissance d'une culture liée avec la langue concernée. «Pour ce faire il faut définir les concepts de base nécessaires à la production d'un enseignement de langues étrangères, qui permettent à l'apprenant d'intégrer une nouvelle langue ainsi qu'" une nouvelle culture au moyen d'une pédagogie adaptée. » (Byram,2011:256)

Toutes les théories sur le concept de «culture » ne doivent pas perdre de vue la complexité du contexte culturel de diverses sociétés. Il semble que même si de différentes sociétés et cultures sont de plus en plus interconnectées dans un réseau mondial par la diplomatie, le commerce, le tourisme, les échanges des jeunes etc., la capacité à nous adapter culturellement à cette nouvelle dynamique ne progresse pas au même rythme et ne suivent pas toujours la bonne direction; des phénomènes tels que le racisme, la xénophobie, les stéréotypes et les préjugés sont actuellement des générateurs d'innombrables conflits partout dans le monde, d'où l'importance de l'éducation interculturelle pour affronter les tâches actuels et les défis du futurs. Les apprenants des langues étrangères, outre leurs compétences linguistiques, devraient également être dotés de la compétence interculturelle, bien nécessaires pour utiliser la langue de manière appropriée dans une interaction sociale et culturelle.

Aux nouvelles orientations de l'enseignement et de développement de la didactique, la dimension interculturelle est perçu comme l'un des objectifs fondamentaux de l'enseignement des langues étrangères. Beaucoup de recherches se sont effectuées à cet égard en vue de faciliter l'interaction et l'intercompréhension humaine dans le monde qui en pleine mouvement. "Ces recherches fondées sur une prise de position reflètent l'état des relations entre l'enseignement/apprentissage et les conditions sociales où elles sont ancrées ». (Byram, 2011:256)

Ainsi, le développement de la capacité à comprendre les similitudes et les spécificités de différentes cultures devrait être une partie intégrante de l'apprentissage des langues étrangères, ce qui implique la capacité d'interpréter et de comprendre les points de vue des autres, ainsi que de remettre en question leurs propres points de vue. Cela nécessite une plus grande exposition à des contextes de communication authentiques que les matériaux simplement didactiques ne peuvent pas l'offrir. L'enseignement /apprentissage de FLE authentique, étroitement liée à l'interaction active, met l'accent sur l'encouragement des étudiants vers des connaissances et des savoirs bien diversifiés.

Naturellement, pour apprendre une langue étrangère, il faudrait traverser certaines phases de développement linguistique de l'apprentissage qu'on ne peut pas les éviter. Les enseignants ne peuvent pas changer essentiellement le développement du cours linguistique des langues étrangères, mais, ils ont le pouvoir d'influencer de manière significative avec de différentes stratégies d'enseignement au rythme, et par conséquent au degré et à la qualité de l'apprentissage.

L'enseignant ayant comme support les TIC, peut aider à accélérer le processus d'apprentissage, mais pour le faire, il a besoin de construire une perception individuelle claire et complète de cette nouvelle " classe », comme un environnement d'apprentissage riche et illimité dans le temps et dans l'espace et l'appliquer par la

XLinguae, Volume 13 Issue 1, January 2020, ISSN 1337-8384, eISSN 2453-711X 
suite pour ses étudiants. «De plus en plus, l'enseignant devient l'accompagnateur $d u$ processus d'apprentissage (facilitator ou coach), celui qui montre la voie, celui qui motive et qui vient en aide. Il serait évidemment aberrant de penser que l'ordinateur puisse remplacer l'enseignant.» (Desmet, 2006:127)

Un tel environnement se caractérise par l'augmentation de la capacité des apprenants à lire, à écrire et à participer à des discussions de textes oraux et écrits en langue étrangère qui constituent une pratique précieuse pour le développement de l'apprentissage. L'élargissement des capacités à créer, soumettre, exprimer des idées et analyser des textes de différentes typologies favorise la compréhension de la notion du contenu et la familiarisation avec un large éventail de textes. "Il importe maintenant de réaliser un mélange optimal entre c-et e-learning dans chacun des domaines de la didactique des langues, qu'il s'agisse du domaine des connaissances ou de celui des compétences.» (Desmet, 2006:131)

La communication dans des contextes informels, en dehors des murs de la classe est insuffisante pour dépasser un niveau fragile de connaissances et permettre à l'étudiant de devenir un locuteur à part entière d'une langue étrangère. C'est le rôle de l'enseignant grâce à une orientation et implication continue des apprenant à des activités linguistiques d'aider les étudiants devenir des utilisateurs compétents de la langue française, vu que les structures grammaticales de la langue apparaissent systématiquement dans leurs communications en ligne et de construire des activités d'enseignement axées sur une utilisation productive orale/écrite continue de la langue, un élément stratégique sans lequel les autres éléments seraient dévalorisés.

La participation interactive des étudiants, accélérant l'apprentissage des langues étrangères, est la clé de la réussite, à l'opposé de l'apprentissage passif qui l'entrave. Les apprenants impliqués dans la discussion, en vue de découvrir le sens, font des modifications à être " plus accessible ", donc plus compréhensible par les interlocuteurs, ils ont besoin de rétroaction corrective à être capable à améliorer leur performance linguistique. Lors de l'interaction active, ils enrichissent les connaissances linguistiques acquises dans cet nouvel environnement, en accentuant l'attention sur les structures connues et confirmant les doutes et les hypothèses soulevés. Par conséquent, les étudiants parviennent à intérioriser de nouvelles connaissances de langue pour progresser vers un discours approprié et tout en développant un style individuel d'expression.

L'apprentissage des langues étrangères dans cet nouvel environnement d'enseignement, en classe et en ligne, est également lié à l'attitude envers l'erreur. Dans ce contexte, nous devons affirmer qu'il est vrai que les erreurs éventuelles des étudiants ne doivent pas être perçues avec un regard punitif et décourageant, mais en revanche, le non-respect ou l'évitement systématique de la correction des erreurs causerait de graves conséquences sur l'apprentissage. Les erreurs doivent être identifiées et corrigées pour augmenter et préciser la perception sur la façon dont la langue fonctionne, mais sans couper le cours de la conversation, à travers les moyens créatifs, sans être étiquetés comme tels, incitant ainsi une réflexion sur les conventions et les utilisations de la langue. " $\grave{A}$ ce stade, il convient de souligner un certain nombre de stratégies, de la rétroaction corrective, commençons de la correction directe, des demandes de clarification, des répétitions de la phrase, des questions évocatrices ou la clé métalinguistiq ue.» (Desmet, 2006:133) Connaître et utiliser ces techniques réside dans le fait qu'elles sont considérées comme une incitation et pas du tout répressives dans la conception des attitudes des apprenants à l'égard de leurs propres erreurs et des erreurs des autres.

L'enseignant doit planifier avec précision les activités quotidiennes en maintenant et en promouvant les principes d'un nouveau cadre de la notion de classe contemporaine en tant qu'un riche environnement d'apprentissage qui est capable de dépasser les limites de temps et d'espace d'un c-learning. Il devait non seulement analyser et programmer le travail en détail, compte tenu des objectifs, le rôle des 
participants, des nouveaux outils disponibles pour soutenir l'apprentissage au moyen des TIC, mais aussi prévoir des situations difficiles qui peuvent survenir. "Plus que jamais, l'enseignement des langues a besoin de professeurs enthousiastes pour encadrer et assurer le suivi des tâches faisant appel aux TIC.» (Desmet, 2006:129)

L'actualisation et la réalisation de ce processus complexe accompagné des difficultés associées à l'utilisation des TIC et de l' accès à Internet a besoin d'enseignants dotés de nouvelles compétences informatiques. "En effet, le corps enseignant doit adopter les technologies ; il doit accepter de reconnaître leur potentiel, mais aussi leurs limites.» ( Peter-Lippman, 2010:1)

\section{Méthodologie de l'expérimentation :}

\subsection{Informations générales :}

Pour confirmer l'hypothèse énoncée, on a soumis ce type d'enseignement dans les deux environnements d'enseignement /apprentissage à une expérimentation pendant 27 semaines.

Au moyen de trois questionnaires, on s'est adressé aux étudiants, au début et à la fin de l'expérimentation, afin de recueillir leurs opinions et leurs perceptions concernant la contribution des TICE à l'apprentissage de FLE. Un total de 97 étudiants débutants soumis à un apprentissage intensif du français vers le niveau B1, futurs enseignants de FLE en première et deuxième année de l'Université de Tirana, sont impliqués à cette expérimentation d'enseignement/apprentissage, un environnement hybride; en classe et en ligne, ayant comme support les TIC, pendant deux sessions. A travers de la $1^{\text {ère }}$ enquête on a mesuré la fréquence d'accès à Internet; l'utilisation des TIC et son impact comme extra outil à l'apprentissage de FLE. Au moyen du $2^{\text {eme }}$ questionnaire adressé aux étudiants, nous avons mené une étude quantitative et comparative pour prendre connaissance sur : des informations générales; la situation réelle de leur expérience informatique; l'intégration des TIC dans leur vie; le niveau de la compétence informatique; l'impact des TIC à leur apprentissage; pour continuer avec des questions d'autoévaluations de leurs compétences informatiques à chercher de l'information sur Internet; et enfin sur les sujets qui leur intéressent.

A la fin de l'expérimentation, par le $3^{\text {eme }}$ questionnaire les étudiants ont évalué le potentiel de ce type d'enseignement, le degrés de la motivation et d'insécurité au début et à la fin des activités.

\subsection{Modèle d'un mini-projet en utilisant les TICE}

Thème: Sauver la planète

Objectif général: Savoir identifier les problèmes écologiques et réagir trouvant des solutions à travers la communication en ligne.

Public: Etudiants de la filière du français: niveau débutant.

Objectifs:

$\square$ Savoir exprimer les problèmes variés sur l'écologique

Développer des compétences communicatives dans le domaine de l'écologie à travers:

-la lecture collectant des données sur le site

-l'écriture à travers les productions écrites en ligne

-le parler à travers des débats et des présentations.

$\square$ Elargir le lexique des apprenant dans ce domaine:

Par exemple:

- agir $=$ faire quelque chose

-GIEC : Groupe d'experts intergouvernemental sur l'évolution du climat

-faire grève $=$ ne pas aller travailler

XLinguae, Volume 13 Issue 1, January 2020, ISSN 1337-8384, eISSN 2453-711X 
-l'environnement $=$ la nature

-l'inaction $=$ absence d'activité

-le soutien = le support

-le renouvellement $=$ le rénovation

-Les gaz à effet de serre (GES) sont des composants gazeux qui absorbent le rayonnement infrarouge émis par la surface terrestre et contribuent ainsi au réchauffement climatique.

-Le covoiturage est l'utilisation conjointe et organisée d'une voiture automobile, par un conducteur non professionnel et un ou plusieurs passagers, dans le but d'effectuer un trajet commun.

- $5 G$ est la cinquième génération de standards pour la téléphonie mobile

Renforcer les différentes structures de langue de faire par exemple des questions en français.

Encourager une réflexion critique et créative à un travail de groupe en intégrant la messagerie électronique et les groupes en ligne.

\subsection{Description du processus :}

On propose une page Web et on envoie du matériel pédagogique qui parle des problèmes de l'écologique. Avec une recherche Google sur les problèmes de l'écologie pour trouver de nombreuses adresses comme support de recherches. Chaque étudiant définit une page avec certains priorités. Les étudiants du groupe visitent ces sites Web, lisent des informations disponibles pour développer les principaux problèmes qui concernent l'environnement, en cherchant des informations plus détaillées sur les problèmes écologiques, les risques et les impacts sur l'environnement et la vie humaine en particulier, ainsi que du matériel promotionnel. Le professeur vérifie ces productions pour évaluer le degré de précision à propos de la question concernée.

Les étudiants préparent une présentation orale pour partager avec le reste du groupe ce qu'ils ont découvert sur l'environnement et pour présenter le matériel promotionnel qu'ils ont recueilli.

Ensuite, un espace est prévu à ce stade pour des activités extracurriculaires, mis à jour au moyen d'un débat ouvert avec l'auditeur.

De plus, afin de valoriser le travail créé, les présentations sont publiées en ligne sur le site Web du groupe, afin de sensibiliser un public plus large, au dehors de la salle de classe, à l'utilisation la plus raisonnable et économique des ressources énergétiques.

A la fin de chaque activité, il faut évaluer les étudiants, en vue de les encourager à continuer à utiliser les TICE en fonction de l'apprentissage du FLE.

\subsection{Résultats et discussion}

$\mathrm{Au}$ moyen d'une méthodes quantitatives à caractère analytique et comparatives des données recueillies à travers l'évaluation, l'autoévaluation et l'observation personnelle/professionnelle, on a conclu à ces résultats :

D'abord, à travers de la $1^{\text {ère }}$ enquête et par une analyse quantitative résulte que :

Il ont été impliqués 97 étudiants ; dont 29 garçons et 68 filles, âgés de18 à 21 ans, le niveau de français vers B1.

Quant à l'équipement, les données montrent que des apprenants sont suffisamment équipés en ordinateurs : $99 \%$ des étudiants possèdent des ordinateurs personnels, $1 \%$ disposent d'au moins un ordinateur à l'université dans la salle d'informatique. Ils passent de 2 à 5 heures devant l'ordinateur qui est presque toujours connecté, ayant de bonnes compétences concernant certaines applications informatiques tel que le chat, les blogs, les programmes de présentation(power point) etc. Ils communiquent fréquemment entre eux par SMS, par téléphone et par chat ; rarement par mail. Ils n'utilisent pas beaucoup les forums de discussion. 
Environ $60 \%$ du temps passé en ligne est utilisé pour communiquer, 20\% pour s'informer, $20 \%$ pour se divertir et $10 \%$ pour des questions linguistiques.Presque tous les étudiants avaient l'habitude d'utiliser Google, Wikipédia etc. en fonction de l'apprentissage. Les principaux sujets qui leur intéressent à traiter au cours de langue étaient: l'écologie, les traditions, le marché du travail, le tourisme, la discrimination/racisme, la musique etc. Par les données recueillies du $2^{\text {ème }}$ questionnaire on a les résultats suivants : tous les étudiants impliqués à l'expérimentation ont répondu aux questionnaires ; l'accès à Internet soit 100\%; 97\% des apprenants utilisent des TIC comme extra outil à l'apprentissage; 99\% des étudiants pensent que les TIC ont une contribution positive à l'apprentissage. Les résultats globaux de cette enquête ont révélé des perspectives positives à l'utilisation des TIC dans l'apprentissage des langues étrangères en générale et particulièrement du FLE. D'après l'analyse des résultats du $3^{\text {eme }}$ questionnaire, les étudiants ont apprécié positivement le potentiel de ce type d'enseignement, ils se sont sentis à l'aise et impliqués pendant les activités et l'insécurité a presque disparu à la fin de l'expérimentation faisant preuve d'une haute motivation pour un apprentissage autonome et durable. Malgré les obstacles et les limites présents, les résultats de l'analyse des commentaires des étudiants peuvent être interprétés de manière cohérente comme une preuve de contribution positive des TIC à l'apprentissage du français authentique.

\section{Conclusion}

L'analyse comparative et l'observation personnelle/professionnelle nous amènent à conclure que les TIC en multipliant le degré et la qualité d'exposition à de nombreuses contextes linguistiques authentiques, où les éléments socio-culturels et interculturels sont naturellement inclus, soutiennent fortement le processus de l'enseignement /apprentissage de FLE authentique en vue d'une interaction réussie. Ce type d'apprentissage participatif et collaboratif ne se réduit pas à la mémorisation et reproduction des informations facilement disponibles, mais il développe la capacité à comprendre et agir en fonction de la résolution des problèmes spécifiques de la société où l'apprenant fait partie en tant qu'individu. Les discussions des étudiants manifestent une réflexions critique et une pluralité des points de vue témoignant d'un développement de la pensée critique autonome. $\mathrm{Vu}$ que chaque public a ses propres intérêts bien particuliers, chaque enseignant peut adopter un modèle propre à lui afin d'assurer du succès dans l'enseignement/apprentissage dans ce nouveau contexte sociolinguistique en présence des TICE.

Les nouvelles représentations des enseignants et des apprenants, font partie importante des conditions essentielles à la réussite du processus éducatifs pour une intercompréhension entre francophones aux échelles régionale et internationale.

\section{Bibliographic references}

AYCAN, A. 2013. L'enseignement du FLE aux enfants assisté par le multimédia, in "Turkish Studies": International Periodical For The Languages, Literature and History of Turkish or Turkic, Ankara, VIII, n.10, 87 p.

BANGE, P. 1996. Considérations sur le rôle de l'interaction dans l'acquisition d'une langue étrangère Les carnets du Cediscor, $189 \mathrm{p}$.

BLAKE, R J. 2008. Brave New Digital Classroom -Technology and Foreign Language Learning, Washington, DC: Georgetown University Press, 19 p.

BIGGS, J.L. 1997. L'apprentissage en semi-autonomie en anglais: la place de nouvelles technologies in Cahiers de l'APLIUT, $10 \mathrm{p}$. 
BYRAM, M. 2011. La compétence interculturelle Blanchet, Ph., Chardenet, P., Guide pour la recherche en didactique des langues et des cultures Éditions des archives contemporaines (EAC), $256 \mathrm{p}$.

CONSEIL DE L'EUROPE. 2001. Cadre européen commun de référence pour les langues : apprendre, enseigner, évaluer, Paris, Edition Didier, 16 p.

DESMET, P. 2006/1. L'enseignement/apprentissage des langues à l'ère du numérique: tendances récentes et défis. Revue française de linguistique appliquée, vol. XI, pp. 119,124,126,129,131

HIRSCHSPRUNG, N. 2005. Le multimédia in Apprendre et enseigner avec le multimédia. Hachette, Paris, 14 p.

GILBERT, L. - MOORE, D. R. 1998. Building interactivity into Web courses: Tools for social and instructional interaction, Educational Technology, 38/3, 29 p.

LICHERI, L M. 2018. L'apprentissage par projet, méthode pour le développement des compétences du XXI ${ }^{\text {ème }}$ Siècles et la diffusion des valeurs européennes. Partenariat entre les écoles 2015-2017, UE. 11 p.

LIPPMAN, C P. 2010. JCJ Architecture, L'environnement physique peut-il avoir un impact sur l'environnement pédagogique ? New York CELE Échanges 2010/13, OCDE, 1 p.

PERRENOUD, P. 1994. La formation des enseignants entre théorie et pratique. Paris : L'Harmattan, 9 p.

POWELL, K. - KALINA, C. 2009. Cognitive and Social Constructivism: Developing Tools for an Effective Classroom", Education, 130/2, 239 p, 243 p.

TURKLE, Sh.1996. Life on the screen: Identity in the age of the Internet, New York: Touchstone, $12 \mathrm{p}$.

VALENZUELA, O. 2010. La didactique des langues étrangères et les processus d'enseignement /apprentissage in Synergies, n. 6, Chili, 87, 89 p.

Words: 6177

Characters: 43150 (23,97 standard pages)

Dr. Klementina Shiba

Faculté des Langues Etrangères

Université de Tirana

Rue « Elbasani »

Tirana

Albanie

shibaklementina@gmail.com 